\title{
Characterization of Polyurethane Coated Aerospace Aluminium Alloy (7075) By DSC, XRD and Adhesion Test
}

\author{
Venkatakrishnan $\mathbf{R}^{1 *}$, Senthilvelan $\mathrm{T}^{2}$ and Vijayakumar $\mathrm{T}^{3}$
}

${ }^{1}$ Department of Mechanical Engineering, IFET College of Engineering, Villupuram, Tamilnadu, India

${ }^{2}$ Department of Mechanical Engineering, Pondicherry Engineering College, Pillaychavadi, Pondicherry, India

${ }^{3}$ Zeero Solar, 3-A, Defence Colony Road, St Thomas Mount, Chennai, India

\begin{abstract}
The research is envisaging characterizing the Polyurethane (PU) coating on AA 7075 so as to predict the aircraft coating life. Differential Scanning Calorimetry (DSC) and X-ray Diffractometry have been employed to investigate the differences in thermal behavior and crystalline structure of the polyurethane (GB BOND 141). DSC can be used to examine the material from a subambient starting temperature to the glass transition event and finally through the crystalline melting region. X-ray diffraction (XRD) provides important structural information about the polyurethane in the structure of semi-crystalline polymer substance consisting of two phases, viz., amorphous and crystalline as well as their fine texture which has a strong effect on physical and mechanical properties of polyurethane. After analyzing the characteristics, the extent of coating loss is examining visually in order to evaluate the adhesion property before carrying out the peel-off test.
\end{abstract}

Keywords: Polyurethane; Aluminium 7075; DSC; XRD; Peel-off

\section{Introduction}

Economic competitiveness and environmental concerns have driven the coating technologist to explore newer chemistry and approaches to improve the efficiency of organic coatings at a minimum volatile organic component (VOC). Organic coatings or paints on a substrate give aesthetic appearance as well as protection from the destructive phenomenon known as corrosion. Coatings can provide materials with the desired aesthetical properties such as color and gloss, but they are also of vital importance in the protection against environmental influences, including moisture, radiation, biological deterioration or damage from mechanical or chemical origin $[1,2]$.

Polyurethane (PU) coatings opened the door for automotive applications. Formulations and processing techniques continuously developed as one- and two-pack systems were developed. The PU coating industry has entered a stage of stable progress and advanced technological exploitation. Today, PU coatings can be found on many different materials to improve their appearance and lifespan.

On automobiles, PU coatings provide the demanded exterior high gloss, improved color retention, improved scratch and corrosion resistance. Different types of $\mathrm{PU}$ coatings have been applied in construction, where building floors, steel trusses and concrete supports are spray coated to make them more durable against environmental deterioration with minimum cost to maintain $[3,4]$.

The research reported here investigated the importance of the characterization of PU coating on AA7075 to check their thermal stability, structure of PU and adhesion strength.

\section{Experimental Procedure}

\section{Sample preparations}

The coating system used in this study was epoxy polyamide primer (GP GUARD 144) and with a high gloss polyurethane topcoat (GP BOND 141). The coating substrate was AA7075-T6 and had been purchased from Perfect Metal Alloy Company. After cleaning aluminium surface with methyl ethyl ketone, and the coatings were applied by spray painting using a conventional spray gun. The primer film thickness was maintenance approximately in a range of $30 \pm 0.5$ $\mu \mathrm{m}$, while the coat of polyurethane top was in the range of 35-60 \pm $1.5 \mu \mathrm{m}$. The schematic diagram of coating layer of sample is shown in Figure 1 [5].

\section{Coating thickness measurements}

After coating, epoxy polyamide primer layer and polyurethane topcoats on AA7075 surface coating thickness should be measured to achieve the required level. PHYNIX Surfix Probe [6] is type coating thickness gauge was used to measure the coating thickness level of each primer and topcoat layer on the aluminium surface.

\section{Characterization}

Differential scanning calorimetry (DSC) measurements were conducted using a thermal analyzer (DSCQ20 V24.2 Build 107, TA

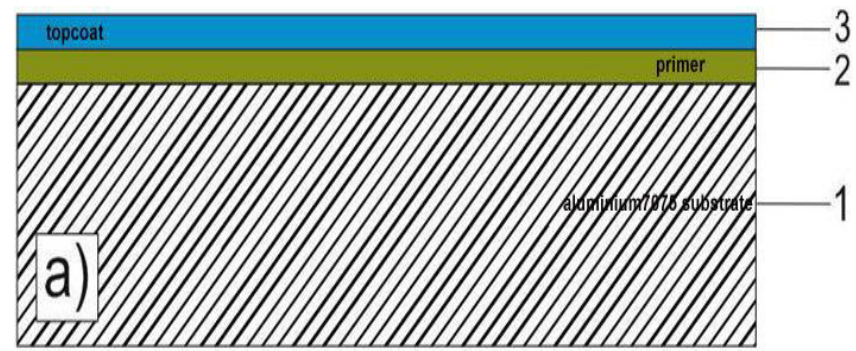

Figure 1: The actual coating layers 1) AA7075 substrate 2) Epoxy polyamide as primer layer 3) Polyurethane as topcoat layer.

*Corresponding author: Venkatakrishnan R, Assistant Professor, Department of Mechanical Engineering, IFET College of Engineering, Villupuram, Tamilnadu, India-605 108, Tel: 04146231 456; E-mail: venkatmech105@gmail.com

Received November 20, 2016; Accepted December 11, 2017; Published December 14, 2017

Citation: Venkatakrishnan R, Senthilvelan T, Vijayakumar T (2017) Characterization of Polyurethane Coated Aerospace Aluminium Alloy (7075) By DSC, XRD and Adhesion Test. J Appl Mech Eng 6: 296. doi: 10.4172/2168-9873.1000296

Copyright: () 2017 Venkatakrishnan R, et al. This is an open-access article distributed under the terms of the Creative Commons Attribution License, which permits unrestricted use, distribution, and reproduction in any medium, provided the original author and source are credited. 
Citation: Venkatakrishnan R, Senthilvelan T, Vijayakumar T (2017) Characterization of Polyurethane Coated Aerospace Aluminium Alloy (7075) By DSC, XRD and Adhesion Test. J Appl Mech Eng 6: 296. doi: 10.4172/2168-9873.1000296

Instruments). A polyurethane coated surface was peeled off and made into the powder form of $10 \mathrm{mg}$, which is required level for DSC studies. DSC is used widely for examining polymers to check a number of characteristic properties, such as two major thermal transitions: the glass transition temperature $(\mathrm{Tg})$, the melt temperature( $\mathrm{Tm})$. In the test the temperature of PU sample increased from room temperature to $200-250^{\circ} \mathrm{C}$ with a heating rate of $10^{\circ} \mathrm{C} \mathrm{min}^{-1}$.

A scanning electron microscope (SEM) is a type of electron microscope that images a sample by scanning it with a high-energy beam of electrons in a raster scan pattern. Scanning electron microscope (SEM) studies were performed in a JSM-6610 scanning electron microscope (JEOL/EO). SEM can produce very high-resolution images of a sample surface, revealing details about less than 1 to $5 \mathrm{~nm}$ in size. Due to the very narrow electron beam, SEM micrographs have a large depth of field yielding a characteristic three-dimensional appearance useful for understanding the surface structure of a sample. Therefore, resolutions, magnification and depth of field are important an element of SEM [6].

$\mathrm{X}$-ray diffraction (XRD) provides important structural information for polymers. Useful XRD analysis data was obtained from crystalline, semi-crystalline, amorphous polymeric materials. Significance information of structure can be obtained from peaks in XRD pattern, including peak position, peak shape and peak width as well as peak intensity, to identify crystalline phases and orientation and determine structural properties: $\mathrm{d}$-spacing's and lattice parameters, residual strain, particle or grain size, phase composition, etc [7]. The diffraction profiles show an amorphous broad shoulder, diffused diffraction maximum at $2 \theta=20^{\circ}$. It is likely that some soft segment-hard segment phase mixing could occur in the system disturbing the soft segment crystallization. This may account for the broader diffraction.

\section{Peel off test}

An essential requirement of protective coatings is that they should adhere firmly on the substrate to which they are applied. This is an obvious requirement, but it is a difficult one to measure quantitatively. Many procedures are available for assessing coating adhesion, including ASTM D 3359 for peel off test. The aluminium alloy 7075 sample size is $50 \mathrm{~mm} \times 50 \mathrm{~mm}$ was coated with polyurethane in required microns to measure the strength of polyurethane that they adhere on the substrate.

\section{Results and Discussion}

\section{Differential scanning calorimetry (DSC)}

Differential scanning calorimetry or DSC is a thermo-analytical technique. DSC is used widely for examining polymers to check a number of characteristic properties, such as two major thermal transitions: the glass transition temperature (Tg), the melt temperature( $\mathrm{Tm})$. Figure 2 shows test the temperature of $\mathrm{PU}$ sample increased from room temperature to $150-170^{\circ} \mathrm{C}$ with a heating rate of $10^{\circ} \mathrm{C} \mathrm{min}^{-1}, 20^{\circ} \mathrm{C}$ $\mathrm{min}^{-1}$ and $40^{\circ} \mathrm{C} \mathrm{min}^{-1}$, respectively. Glass transitions may occur as the temperature of an amorphous solid is increased. These transitions appear as a step in the baseline of the recorded DSC signal. This is due to the sample undergoing a change in heat capacity; no formal phase change occurs. From Figure 3 it can be observed that Tg value depends on heating history and is subjected to thermal lag, extrapolation to zero heating rate which yields an accurate value for $\mathrm{Tg}$ and is $112.5^{\circ} \mathrm{C}$ at zero heating rate. This finding was clearly proved by Xin Fu [8]. Figure 3 shows below the relation between glass transition temperature Tg and heating rate. Figure 4 shows the measurements differential scanning calorimetry (DSC) using TA Instrument DSCQ20 V24.2 Build 107

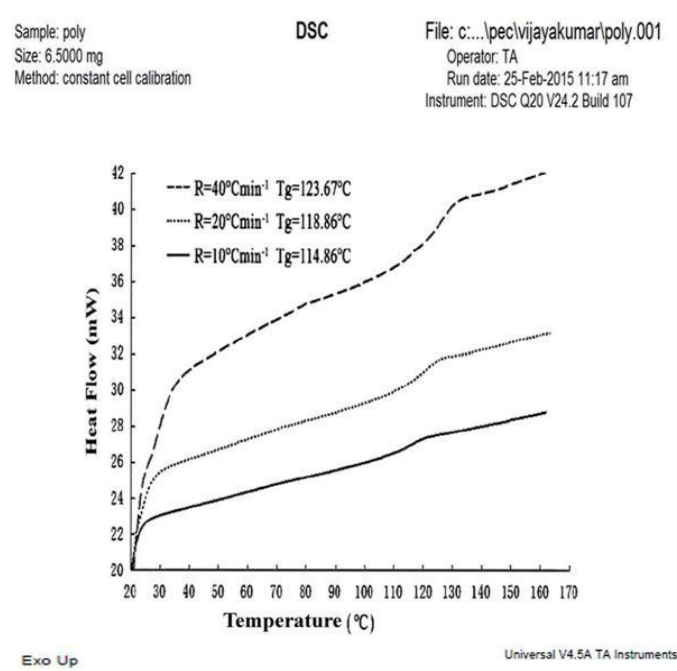

Figure 2: The polyurethane $\mathrm{Tg}$

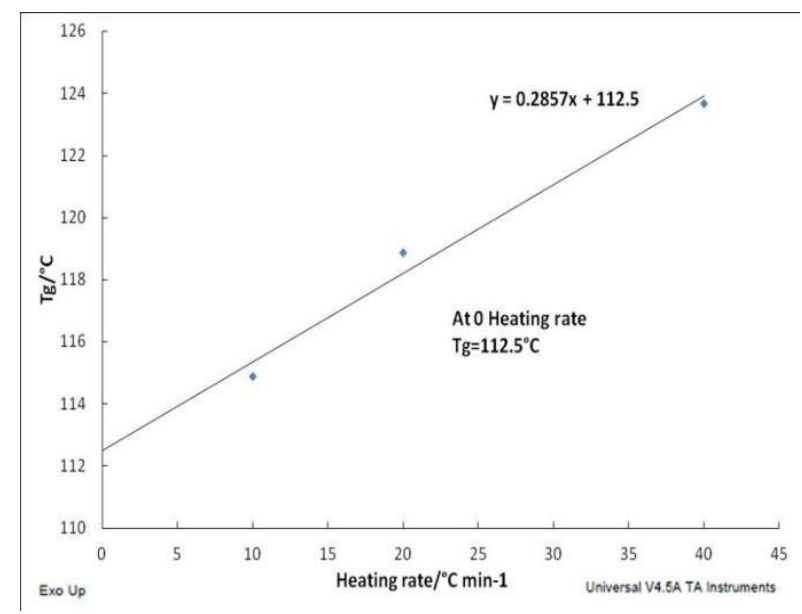

Figure 3: The heating rate of $\mathrm{Tg}$ at $0^{\circ} \mathrm{C}$

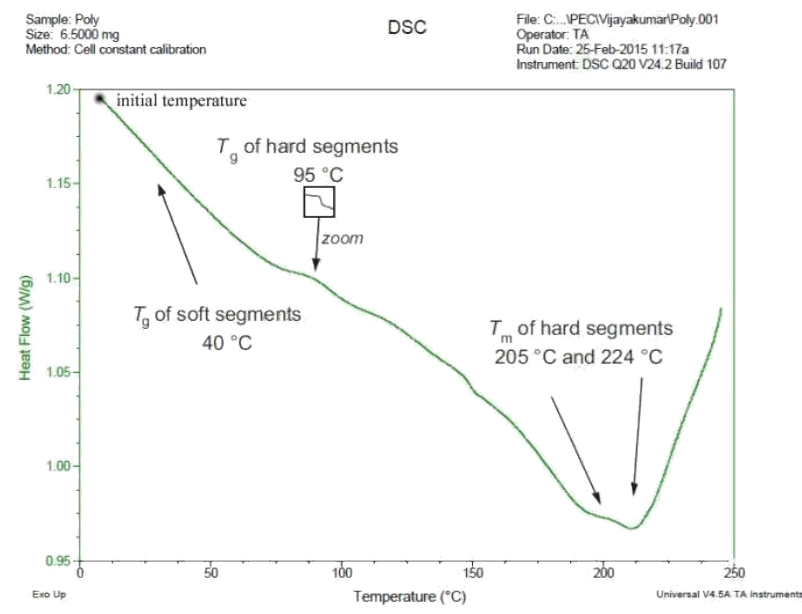

Figure 4: DSC curve of polyurethane (endothermic peaks downwards).

device. Two endothermic peaks are well displayed on the DSC curve associated to two melting temperature $(\mathrm{Tm})$ at $205^{\circ} \mathrm{C}$ and $224^{\circ} \mathrm{C}$. Saiani et al. [9] absorbed that these two endotherms are due to the melting of 


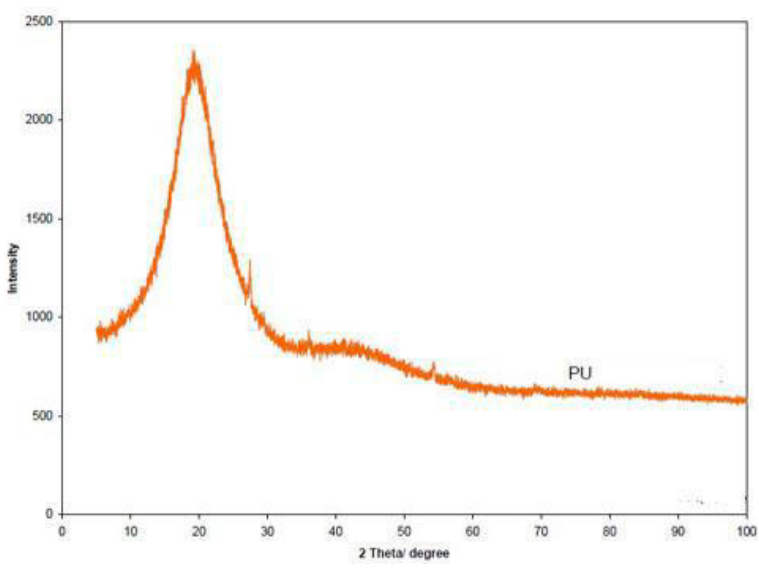

Figure 5: Polyurethane XRD.
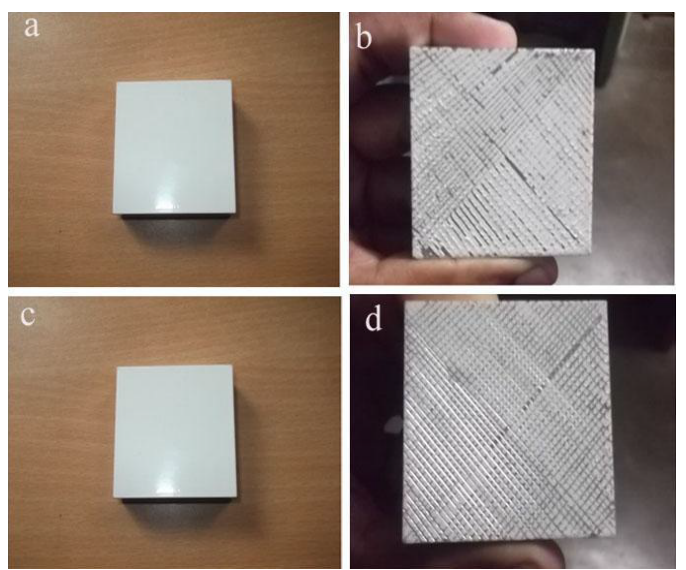

Figure 6: shows peel off test taken a) PU coated in specific microns b) after cut perpendicular line final weight should be measured c) PU coated in specific microns d) after cut perpendicular scribe removed from panel final weight should be measured.

an ordered structure appearing in the hard phase and to a micro phase mixing of soft and hard segments. The DSC curve also shows, a glass transition at the temperature $\mathrm{T}_{\mathrm{g}}^{\mathrm{ss}}$ of approximately $40^{\circ} \mathrm{C}$ related to the soft segments. As described by Barikani and Hepburn [10] and Cuve et al. [11] as mentioned by the PU manufacturer, another glass transition associated to the hard segments appears at the temperature $\mathrm{T}_{\mathrm{g}}^{\mathrm{HS}}$ of $95 \pm$ $1^{\circ} \mathrm{C}$. Considering the DSC curve with the values of glass cand melting temperature, the hard and soft segments content can respectively be estimated [9].

\section{$\mathrm{X}$-ray diffraction (XRD)}

$\mathrm{X}$-ray diffraction (XRD) was carried out for polyurethane samples. $\mathrm{XRD}$ result of polyurethane was shown in Figure 5. As evidenced in Figure 5, the diffraction profiles show an amorphous broad shoulder, diffused diffraction to be maximum at $2 \theta=20^{\circ}$. It is likely that some soft segment-hard segment phase mixing could occur in the system disturbing the soft segment crystallization [12]. This may account for the broader diffraction. For ordinary synthetic polymer, the nonuniformity of the molecules makes it impossible to form perfect single crystals. As a result, amorphous and crystalline phases are present in a real polymer, and these entities have complex organization. Diffraction occurs only when Bragg's Law is satisfied condition for constructive interference from planes with spacing $\mathrm{d}$ to be.
$2 \mathrm{~d} \sin \theta=\mathrm{n} \lambda$

Where $d$ is interplanar spacing, $2 \theta$ is diffraction angle; $\lambda$ is the wavelength of the incident X-ray beam.

\section{Peel off test}

Figures $6 \mathrm{a}$ and $6 \mathrm{c}$ show the specimen which are coated to a thin (80-micron) on aluminium alloy 7075. During preparation for testing, the coated polyurethane on aluminium bar $(50 \mathrm{~mm} \times 50 \mathrm{~mm})$ is cut into strips. Figures $6 \mathrm{~b}$ and $6 \mathrm{~d}$ show the panel is first divided into strips by cutting through the coating to the substrate in several perpendicular lines along the length of the panel and is 50 such perpendicular lines were made in opposite to each other. Now the coated substrate is lined by giving perpendicular to each other and the scribe if fixed on coated panel. Some hand pressure on scribe. After 10 minutes the scribe tape is suddenly peeled off by hand pressure.

Peel adhesion tests on coatings provides a method for obtaining quantitative, repeatable peel strength data. Before cutting lines in the sample, the initial weight and after cutting scribe was removed from the panel. The final weight of the sample was also measured to assess the coating loss. In Figure 6a, initial weight of the sample is $114.400 \mathrm{~g}$ and in Figure $6 \mathrm{~b}$ final weight of the sample is $114.394 \mathrm{~g}$. Figure $6 \mathrm{c}$ shows initial weight of the sample as $114.470 \mathrm{~g}$ and in Figure $6 \mathrm{~d}$ final weight of the sample is $114.470 \mathrm{~g}$. The variation of measurements was less than 10 percent which include coating loss. In the polyurethane coating study, distinctive peel strength zones were easily detected for scribed coatings exposed to immersion environments. The coating substrate shows that polyurethane is having good adhesion between aluminium alloys 7075 . Even though differences on the two samples on the peel strengths were observed on the coatings, the same high adhesion rating has observed when tested according to ASTM D 3359 [13]. For both samples of wet applications, this procedure, contribute to improved understanding of the adherence of polyurethane coatings to aluminium and to improved coating, proceeding formulations.

\section{Conclusion}

The tribological experiments, chemical and thermal analysis characterizations indicated that polyurethane showed a semi crystalline structure and, in particular, a glass transition temperature at $95 \pm 1^{\circ} \mathrm{C}$ associated to the hard-crystalline segments (urethane blocks). XRD shows, the amorphous and crystalline phases that are present in a real polymer and it indicate polyurethane is good polymer for industrial applications.

Conducting peel adhesion tests on coatings provide a method for obtaining quantitative, repeatable peel strength data. The variation of measurements was less than 10 percent. In this polyurethane coating study, distinctive peel strength zones were easily detected for scribed coatings exposed to immersion environments. The coating substrate showed that polyurethane is having good adhesion between aluminium alloys 7075 . For both samples on wet applications, this procedure should have contributed to improve the adherence of polyurethane coatings to aluminium and also to enhance the coating proceeding formulations.

\section{References}

1. Chattopadhyay DK, Raju KVSN (2007) Structural engineering of polyurethane coatings for high performance applications, Organic Coatings and Polymers Division. Polym Sci 32: 352-418.

2. Usmani AM, Donley M (2000) Aircraft-coating weathering studies by analytical methods, ALTEC USA, Indianapolis, Indiana 46278, Air Force Research Laboratory, Wright-Patterson Air Force Base, Ohio. 
Citation: Venkatakrishnan R, Senthilvelan T, Vijayakumar T (2017) Characterization of Polyurethane Coated Aerospace Aluminium Alloy (7075) By DSC, XRD and Adhesion Test. J Appl Mech Eng 6: 296. doi: 10.4172/2168-9873.1000296

3. William GS (2000) The selection, application and inspection of $100 \%$ solids polyurethane coatings for corrosion protection, Ontario L9T 3Y5 Canada.

4. Twite RL, Bierwagen GP (1998) Review of alternatives to chromate for corrosion protection of aluminum aerospace alloys Prog Org Coat 33: 91-100.

5. Danuta K, Przerwa M, Szumiata T (2014) Resistance to erosive wear of epoxypolyurethane coating modified with nanofillers. Chrobrego 45, Radom 26-600, Poland. J Mat Sci Research.

6. Poclain Hydraulics Company (2000) A world of off-road solutions. Pondicherry.

7. Trovati G (2009) Characterization of polyurethane resins by TGA, and XRD. J App Polym Sci.

8. Xin Fu (2012) Active screen plasma surface modification of polymeric materials for biomedical applications. The University of Birmingham, UK.
9. Saiani A (2001) Origin of multiple melting endotherms in a high hard block content polyurethane. Thermodynamic investigation, Macromolecules 34 9059-9068.

10. Barikani M, Hepburn C (1987) Isocyanurate cross-linking as a mean of producing thermally stable polyurethane elastomers. Cell Polym 6: 41-54.

11. Cuvé LJP (1991) Synthesis and properties of polyurethanes based on polyolefine: Rigid polyurethanes and amorphous segmented polyurethanes prepared in polar solvents under homogeneous conditions. Polymer 32: 343352.

12. Kim BK (1996) Polyurethanes having shape memory effects. Polymer 37 5781-5793.

13. Mary E (1995) Measuring of peel adhesion of coating. Fire Research Lab Gaitbemburg, USA. 